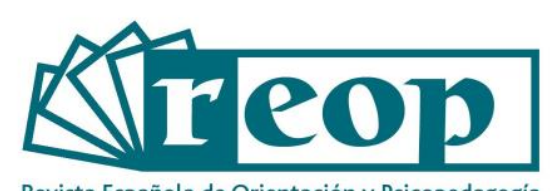

Revista Española de Orientación y Psicopedagogía

\title{
ANÁLISIS DEL PROGRAMA EDUCATIVO “CUBILETE” PARA LA PREVENCIÓN DE ADICCIONES A TIC EN ADOLESCENTES. CASO ESPECÍFICO DE JUEGOS DE AZAR Y APUESTAS ONLINE
}

\section{ANALYSIS OF THE "CUBILETE" EDUCATIONAL PROGRAM FOR THE PREVENTION OF ICT ADDICTIONS IN ADOLESCENTS. SPECIFIC CASE OF GAMBLING AND ONLINE BETTING}

\author{
Beatriz Berrios Aguayo \\ Universidad de Jaén. Facultad de Humanidades y Ciencias de la Educación. Departamento de \\ Pedagogía. Jaén, España \\ Bárbara Pérez García \\ Fernando Sánchez Valenzuela \\ Asociación Ludópatas Jiennenses en Rehabilitación. Jaén, España \\ Antonio Pantoja Vallejo ${ }^{1}$ \\ Universidad de Jaén. Facultad de Humanidades y Ciencias de la Educación. Departamento de \\ Pedagogía. Jaén, España
}




\section{RESUMEN}

El objetivo de este estudio fue analizar la efectividad del programa educativo "Cubilete" para la prevención de adicciones a Internet, móvil, juegos y apuestas online en estudiantes de Educación Secundaria y Bachillerato, diseñado por la Asociación Alujer. La muestra estuvo compuesta por un total de 637 alumnos de diferentes centros de la ciudad de Jaén (España). El instrumento para medir la eficacia del programa en una medida pre y postest fue el Cuestionario escolar de creencias, actitudes y usos de tecnologías, juegos virtuales y apuestas online (CCAU), construido ad hoc. La intervención tuvo una duración de 4 semanas y fue llevada a cabo durante las horas de tutoría. Los resultados muestran una reducción significativa en cuanto al uso de Tecnologías de la Información y la Comunicación (TIC), así como en la frecuencia en las que se utilizan las mismas. También se observó un aumento en el reconocimiento de adicción a las TIC una vez llevada a cabo la intervención, además de un mayor conocimiento de los padres del uso que hacen sus hijos de las mismas. Así mismo, la participación a juegos de azar y apuestas online se ve reducida considerablemente. Por último, hay un esclarecimiento en lo referido al significado del concepto adicción, aunque no significativamente del concepto juego patológico. Como conclusión se destaca el papel revelador que tiene este tipo de intervenciones sobre la población joven en lo referido al uso de las TIC y las adicciones a juegos de azar, virtuales y apuestas.

Palabras clave: Intervención, tutoría, tecnologías de la información y la comunicación, adolescente.

\section{ABSTRACT}

The objective of this study was to analyze the effectiveness of the educational program "Cubilete" for the prevention of addictions to Internet, mobile, games and online bets in students of Secondary Education and Baccalaureate, designed by the Alujer Association. The sample consisted of a total of 637 students from different centers in the city of Jaén (Spain). The instrument to measure the effectiveness of the program in a pre- and post-test measure was the school Cuestionario escolar de creencias, actitudes y usos de tecnologías, juegos virtuales y apuestas online (CCAU), made ad hoc. The intervention lasted 4 weeks and was carried out during the tutorial hours. The results show a significant reduction in the use of Information and Communication Technologies (ICT), as well as in the frequency in which they are used. There was also an increase in the recognition of addiction to ICT once the intervention was carried out, as well as a greater knowledge of the parents of the use made by their children. Likewise, participation in online gambling and betting is considerably reduced. Finally, there is clarification regarding the meaning of the concept of addiction, although not significantly of the concept of pathological gambling. In conclusion, the revealing role of this type of interventions on the young population in the use of ICT and addictions to gambling, virtual and betting is highlighted.

Key Words: Intervention, tutorial hours, information and communication technologies, adolescent.

\section{Introducción}

Cada día son más habituales los problemas educativos derivados de un mal uso de Internet en las poblaciones más vulnerables de la sociedad. En Europa, nueve de cada diez jóvenes de entre 12 y 18 años están actualizados tecnológicamente, lo que provoca que sea más habitual un uso 
abusivo de las tecnologías. Para ser más específicos, de entre el $3 \%$ y el $24 \%$ de los adolescentes hacen un uso abusivo de las TIC (Ólafsson, Livingstone, y Haddon, 2014). Esta nueva situación ha provocado que los padres se vean en la necesidad de indagar acerca de cómo ellos mismos perciben el uso que sus hijos hacen de las mismas, cómo definen su propio papel como agentes de socialización y cómo realizan una mediación mediante la comunicación y la conexión con los adolescentes en relación a este tema (Symons, Ponnet, Walrave, y Heirman, 2017).

Por otra parte, el uso de Internet en dispositivos móviles ha ascendido considerablemente en los últimos años. Para lo que antes se requería una computadora de mesa, ahora se realiza en un aparato tecnológico de no más de $7 \times 7 \mathrm{~cm}$. Toda nuestra vida está supeditada a estos aparatos, desde guardar fechas importantes hasta las tarjetas de crédito. Estos cambios de usabilidad de los dispositivos móviles no sólo han sido visibles en la población adulta, sino que la población adolescente también ha disfrutado de las numerosas posibilidades y facilidades de acceso que proporciona la nueva era digital vía móvil. Ese aumento del uso de dispositivos móviles en la etapa adolescente ha provocado cambios en el proceso de socialización, influyendo en los comportamientos y actitudes de estos (Castellana-Rosell, Sánchez Carbonell, Graner-Jordana, y Beranuy-Fargues, 2007; Salehan y Negahban, 2013). Por ello, es relevante examinar cómo el comportamiento mediado por dispositivos móviles entre los adolescentes, de carácter complejo debido a razones de desarrollo vital y culturales (Yan, 2017), interactúa con las características de los datos sociodemográficos y de acceso al móvil para predecir los niveles de su uso, así como las consecuencias que se pueden producir en los jóvenes dada la excesiva accesibilidad a red (Park, 2015).

A su vez, las conexiones digitales promueven nuevas oportunidades, pero también más riesgos para la salud física (Halen y Guan, 2015; Hysing et al., 2015) y psicológica (Chassiakos, Radesky, Christakis, Moreno, y Cross, 2016; Oberst, Wegmann, Stodt, Brand, y Chamarro, 2017) de los jóvenes. Si nos centramos en la parte psicológica, las adicciones a los diferentes aparatos digitales es una de las causas de mayor preocupación (Ciarrochi et al., 2016). El problema principal recae en que los esfuerzos por parte de la comunidad científica se han centrado principalmente en el examen del concepto de adicción y en la metodología de diagnóstico, antes que en estudios sobre el uso problemático de Internet en grupos de alto riesgo y cómo prevenirlo (Chak y Leung, 2004). Además, la generalización en el uso de la palabra adicción, entendida únicamente como el consumo de drogas, ha reducido sustancialmente el campo de investigación. El concepto adicción tiene que ser estudiado como una falta de control ante una determinada conducta, pudiendo ser ésta cualquiera del día a día de la persona (Cía, 2013).

Dentro de las numerosas actividades que pueden provocar conductas adictivas se encuentran las derivadas del uso de las TIC los avances multimedia en el sector de la telefonía móvil, las redes sociales y la difusión de publicidad que realizan los operadores de juegos online (Uchuypoma Canchumani, 2017). El uso de la red para el acceso a juegos de azar o apuestas online ha estado durante los últimos años en el punto de mira principalmente como responsable de conductas de riesgo en adolescentes (Ruiz-Olivares, Lucena, Pino, y Herruzo, 2010). Es en esta etapa donde se presentan problemáticas por abuso en ciertas conductas dado que los sistemas de recompensa y regulación emocional no se encuentran ajustados aun (Pérez de Albéniz-Garrote, Medina Gómeza, y Rubio Rubio, 2019). Estas actividades de forma controlada son socialmente aceptadas, ya que conceden un placer inmediato. Sin embargo, cuando llegan a ser un problema, dada la frecuencia excesiva que la persona realiza de ellas o la dependencia hacia las mismas, esto se convierte en una adicción conductual o también llamado en este caso juego patológico (Ruiz-Olivares et al., 2010; Potenza, 2013). Pero ¿a qué se refiere de forma más explícita el concepto de juego patológico? Este término se define como la actividad de ocio cuyo fin último es obtener beneficios principalmente económicos a partir de apuestas basadas en el azar (Ladouceur, Sylvain, Boutin, y Doucet, 2002). Debido a que se trata de un neologismo, es a partir de 2013 cuando se incluyó el juego patológico en la quinta sección del Manual Diagnóstico y Estadístico de los Trastornos Mentales o DSM V (Carbonell, 2014), siendo éste uno de los manuales más utilizados y mundialmente conocidos en el mundo. De este modo, el juego patológico fue clasificado como un "trastorno de control de impulso" (Rizeanu, 2014), lo que 
supuso un gran paso en cuanto al desarrollo del conocimiento científico ligado a los avances tecnológicos y las consecuencias de un mal uso de las TIC (Pontes y Griffiths, 2015).

En la población joven, la adicción al juego ha sido relacionada con la expectativa de conseguir estados anímicos positivos o aliviar los negativos que comúnmente se experimentan en esta etapa vital (Estévez Gutiérrez, Herrero Fernández, Sarabia Gonzalvo, y Jáuregui Bilbao, 2014). En los últimos años se ha percibido un incremento notable en la participación abusiva del juego en los jóvenes (Delfabbro, King, y Derevensky, 2016). Para ser más exactos, en Europa, un total del $22,6 \%$ de los estudiantes del último año de Educación Secundaria participaron en juegos con apuestas (el 16,2\% en línea).

A pesar de las intervenciones exitosas para la reducción de la participación en juegos de azar y apuestas online en la edad adulta (Ellison, Vale, y Ladouceur, 2017; Garcia-Caballero et al., 2018; Toneatto, 2016), así como el evidente crecimiento de la participación de los adolescentes en este tipo de actividades, pocos han sido los programas de intervención que han tratado de prevenir o paliar ese tipo de conductas (Donati, Primi, y Chiesi, 2014; Walther, Hanewinkel, y Morgenstern, 2013), y otros han considerado que no son efectivas este tipo de intervenciones (Allami y Vitaro, 2015). Cabe destacar la revisión sistemática de Dowling, Merkouris, y Lorains (2016) que determina que sigue encontrándose un vacío científico y una escasa calidad en las intervenciones en contextos reales en relación con la adicción al uso de Internet y al juego en adolescentes. Esto pone de manifiesto que todo intento de reducir el cada vez mayor número de casos de adolescentes que sufren adicciones de ese tipo, es poco. Por tanto, es evidente que se necesitan más investigaciones en relación a la prevención de la adicción, tanto al uso de las TIC como al juego de azar, que permitan establecer marcadores conductuales, así como el desarrollo de algoritmos basados en el comportamiento capaces de predecir la presencia y/o la aparición de este tipo de adicciones (Gray, LaPlante, y Shaffer, 2012). Además, destaca la necesidad de programas educativos de intervención presenciales o en línea (Gainsbury y Blaszczynski, 2011) que de manera conjunta a los proyectos de organización escolar, principalmente en la Educación Secundaria, prevengan estos tipos de trastornos. Y es que programas de orientación educativa, han manifestado resultados positivos en este tipo de problemática (Caro Amada y Plaza de la Hoz, 2016; Rodríguez Torres, Prats, Oberst, y Carbonell, 2018).

Atendiendo al reducido número de intervenciones eficientes observadas en la literatura dentro de los contextos escolares, el objetivo de este estudio es aplicar y evaluar el programa educativo de intervención "Cubilete" para la prevención de adicciones a Internet, móvil, juegos y apuestas online en la población adolescente de distintos municipios de la provincia de Jaén. De este modo se pretendía dar respuesta a la problemática planteada a través de la orientación educativa.

\section{Método}

\section{Diseño}

Se trata de una investigación evaluativa, con un diseño de pretest-postest para comprobar la eficacia del programa de prevención "Cubilete" para combatir las adicciones a Internet, móvil, juegos y apuestas online. El programa en sí actúa como variable independiente, siendo las dependientes aquellas que guardan relación con las adicciones vinculadas con las TIC, tales como el reconocimiento de los participantes sobre esas adicciones, usos dados a tecnologías conectadas a red, participación en juegos de azar, virtuales y apuestas, y conocimiento de 
terminología dentro de este campo de conocimiento, principalmente. Pero también se tuvo en cuenta la variable dependiente juego patológico.

\section{Muestra}

El estudio estuvo compuesto por un total de 637 estudiantes, de los cuales 298 eran mujeres y 339 hombres de Educación Secundaria y Bachillerato de diferentes centros de la provincia de Jaén (España). Los estudiantes participantes en el estudio pertenecían a diferentes niveles académicos ( $1^{\circ} \mathrm{ESO}, \mathrm{n}=89 ; 2^{\circ} \mathrm{ESO}, \mathrm{n}=211$; $3^{\circ} \mathrm{ESO}, \mathrm{n}=166 ; 4^{\circ} \mathrm{ESO}, \mathrm{n}=144 ; 1^{\circ}$ Bachiller, $\mathrm{n}=27$ ).

La muestra se obtuvo por conveniencia, buscando la representatividad de toda la provincia, para lo cual se constituyeron tres estratos: centros de municipios de menos de 10.000 habitantes (dos centros) en los que se impartía una enseñanza más rural, centros de municipios de entre 10.000 y 30.000 habitantes (cuatro centros) caracterizado por ser municipios medianos y un centro de más de 100.000 habitantes. Finalmente, participó en el programa un 97,25\% de la muestra total. La Tabla 1 muestra información sociodemográfica de interés para el estudio.

\section{Tabla 1}

Características sociodemográficas de los participantes

\begin{tabular}{|c|c|c|}
\hline Variables & $\mathbf{F}$ & $\%$ \\
\hline \multicolumn{3}{|l|}{ Tipo de familia } \\
\hline Pareja de hecho o novio & 22 & 3.4 \\
\hline Casados & 505 & 79 \\
\hline Segundas nupcias & 8 & 1.3 \\
\hline Separados o divorciados & 89 & 13,9 \\
\hline Viudo/a & 8 & 1.3 \\
\hline Otros & 5 & 0,8 \\
\hline Total & 637 & 100,0 \\
\hline \multicolumn{3}{|l|}{ Estado actual de la madre } \\
\hline Tareas de la casa & 196 & 30.3 \\
\hline Trabaja & 387 & 60.5 \\
\hline En paro & 38 & 5.9 \\
\hline Jubilada & 9 & 1.4 \\
\hline Otros & 6 & 0.9 \\
\hline Total & 637 & 100.0 \\
\hline \multicolumn{3}{|l|}{ Estado actual del padre } \\
\hline Tareas de la casa & 10 & 1.6 \\
\hline Trabaja & 549 & 86.3 \\
\hline En paro & 36 & 5.6 \\
\hline Jubilado & 22 & 3.4 \\
\hline Otros & 20 & 3.1 \\
\hline Total & 637 & 100.0 \\
\hline
\end{tabular}

Se siguieron las normas de la Declaración de Helsinki (versión 2013) para completar esta investigación en base a la legalidad vigente. Además, anteriormente el Comité de Ética de la Universidad de Jaén (España) aprobó el estudio.

\section{Instrumentos}


El instrumento utilizado fue el "Cuestionario escolar de creencias, actitudes y uso de tecnología, juegos virtuales y apuestas (CCAU) "construido ad hoc para esta investigación. En su estructura inicial, cuenta con preguntas sobre aspectos sociodemográficos, seguidas de cuestiones del tipo: en qué gastan el dinero recibido, frecuencia y tipo de uso de Internet y teléfono móvil, periodicidad de juegos de azar, virtuales o apuestas, conocimiento de los padres del tipo de uso del ordenador y móvil, reconocimiento propio de una frecuencia adecuada o no del uso de Internet y móvil y preguntas sobre la intervención por realizar o realizada. La versión inicial del cuestionario se confeccionó por parte del equipo investigador, tomando como referencia las necesidades detectadas a lo largo de los años anteriores, la experiencia y la literatura existente sobre la temática. En lo relacionado con el uso abusivo de las TIC, el equipo se basó en el "Cuestionario de Experiencias Relacionadas con Internet (CERI)" y el "Cuestionario de Experiencias Relacionadas con el Móvil (CERM) para adolescentes" (Beranuy, Chamarro, Graner, y Carbonell, 2009). En lo referente a la participación en juegos de azar y apuestas online, la redacción de los ítems tomaron como referencia la "Encuesta Europea ESPAD" (Group, 2016). Tras varias revisiones y depuración del banco de items, se construyó una prueba de 18 items de los cuales 4 tienen respuesta dicotómica y 14 categórica con diferente número de opciones de respuesta.

El cuestionario inicial se somete para su validación de contenido al juicio de 10 jueces expertos (5 hombres y 5 mujeres), tomados al azar de contextos donde el juego forma parte de su trabajo cotidiano. En concreto, de los ámbitos educativo y de la psicología social, consiguiendo de este modo valoraciones fidedignas sobre el tema tratado en el cuestionario. En el plazo de una semana de intervalo valoraron el grado de pertinencia de cada ítem en una escala del 1 al 10 (1=deficiente; 10=excelente). Una vez recogidas las respuestas, se reunió de nuevo el equipo investigador principal para analizar las mismas, comprobándose frecuencias, porcentajes, medias y desviación típica de la concordancia con el constructo y con la pertinencia del ítem a la dimensión evaluada, para lo que se utilizó el paquete estadístico SPSS v. 22. Se eliminaron aquellos items que no obtuvieron al menos un $75 \%$ de unanimidad y que correspondían con medias cercanas o por debajo de 9 . Seguidamente, se realizó la prueba $W$ de Kendall, con la finalidad de conocer el grado de asociación entre $\mathrm{k}$ conjuntos de rangos, en este caso, la concordancia entre las respuestas de los jueces, valorando las respuestas sin eliminar y eliminando los items mencionados anteriormente. De forma global, el coeficiente $W$ fue igual a ,200 no siendo significativo $x^{2}(1)=2,000 p \leq, 157$, lo que indica que hay un adecuado índice de concordancia entre los jueces. El análisis de los rangos promedio muestra grandes diferencias entre los mismos, lo que confiere una alta afinidad entre los jueces.

\section{Intervención}

La Asociación de Ludópatas Jiennenses en Rehabilitación (Alujer) fue la encargada del diseño del programa educativo "Cubilete" además de la puesta en marcha del mismo en los diferentes centros escolares. Las personas a cargo de su ejecución fueron psicólogos especializados en adicciones y trastornos compulsivos con la presencia y colaboración del tutor.

La intervención fue realizada a lo largo de cuatro sesiones de 50 minutos de duración cada una repartidas en cuatro semanas en las horas de tutoría de cada grupo-clase. En las dos primeras sesiones se realizaron charlas dirigidas por psicólogos especialistas en la temática, además de presentar vídeos de casos reales con el fin de concienciar a los participantes sobre los riesgos del uso abusivo de las TIC, los juegos online y apuestas virtuales. Los principales temas tratados en las actividades desarrolladas en estas dos primeras sesiones fueron:

- Riesgos y consecuencias de abusar de las TIC.

- Riesgos de realizar un mal uso de las TIC. 
- Información sobre terminología acerca de adicciones al juego de azar y online.

- Sintomatología que puede derivarse de la adicción al juego de azar online

- Riesgos del abuso al juego de azar online.

En las dos siguientes sesiones se desarrollaron actividades participativas entre el equipo investigador, encargado de la ejecución del programa, y el alumnado. Los puntos abordados en las actividades fueron:

- Afianzamiento de los contenidos explicados en las dos primeras sesiones

- Recomendaciones para la prevención del juego de azar

- Recomendaciones para el buen uso de nuevas tecnologías e internet.

- Alternativas al juego de azar y uso de nuevas tecnologías

- Recomendaciones de dedicación de tiempo libre.

La metodología usada en el programa de intervención fue flexible permitiendo, al mismo tiempo, observar las conductas del alumnado. Además, esta flexibilidad hizo posible la adaptación a las características particulares de los estudiantes, ya que se expusieron los contenidos de forma oral para dinamizar la participación, poder abrir debate y conocer así sus opiniones y valoraciones.

El cuestionario CCAU fue cumplimentado por el alumnado antes y después del programa de intervención para evaluar el efecto del mismo en la muestra seleccionada.

\section{Procedimiento}

En primer lugar, se mantuvo un primer contacto con el equipo directivo de los diferentes centros educativos para confirmar su participación en el programa educativo "Cubilete". Una vez aceptada la participación, se conversó con los tutores encargados de los grupos-clases que participarían en el estudio y así adaptarnos a sus programaciones. Seguidamente, le fue entregado al alumnado el consentimiento informado para que fuera firmado por los padres de los alumnos que voluntariamente quisieran participar en el programa. Posteriormente, se les entregó al alumnado el cuestionario CCAU para ser rellenado antes del comienzo el programa. Previamente a dar inicio al programa de intervención, se le detallaron al alumnado los aspectos a considerar sobre el mismo. Tras las cuatro semanas de implementación del programa, nuevamente se les entregó a los participantes el cuestionario CCAU para ver el efecto que el programa había tenido en ellos. Finalmente, los datos resultantes del estudio fueron analizados y comunicados a cada centro escolar para que sirvieran de precedente a futuras investigaciones llevadas a cabo en dichos contextos.

\section{Análisis de datos}

Los datos se analizaron utilizando el programa estadístico SPSS v. 22.0 para Windows (SPSS Inc, Chicago, EE. UU.). El nivel de significación se estableció en $p \leq 0.05$. Se utilizaron frecuencias y porcentajes, además de la prueba Chi cuadrado ( $\mathrm{x} 2$ ), para determinar la asiduidad de las respuestas de los participantes, para el análisis descriptivo de los datos y para determinar la significatividad entre el pre-postest en las diferentes variables del cuestionario. 


\section{Resultados}

Inicialmente, se analizaron las relaciones existentes entre las características sociodemográficas familiares y la frecuencia con la que el alumnado hace uso de las TIC reflejado en la evaluación pretest del cuestionario. De esta forma se ha podido demostrar la asociación entre el tipo de familia y la frecuencia en el uso de las TIC de los estudiantes $\left(X^{2}=64.63 ; p \leq .002\right)$, observada en mayor medida en las familias en las que los cónyuges están casados y/o separados y cuyos hijos usan las TIC todos los días 5 horas o más. Del mismo modo, existe una asociación entre el estado actual de desempeño laboral de la madre y la frecuencia de las TIC $\left(X^{2}=177.65 ; p \leq .001\right)$ y del estado laboral del padre y esa misma frecuencia $\left(X^{2}=87,25 ; p \leq .001\right)$. Esto subraya la importancia que supone el hecho de atender a las variables sociodemográficas para entender el porqué de un crecimiento en la frecuencia del uso de las TIC.

En cuanto a las diferencias significativas localizadas entre el pretest y el postest de acuerdo a las variables analizadas, los resultados muestran que los gastos realizados por los participantes experimentan una reducción significativa del consumo general del alumnado después de la intervención en lo referido al uso de ordenadores, aplicaciones para el móvil, juegos y apuestas online, máquinas tragaperras, etc. $(x 2=333.23, p<.05)$. Por otra parte, el gasto mensual producido por el móvil se ve también reducido demostrando así una posible concienciación en los participantes en el derroche económico en lo referido a las TIC $(x 2=116.57, p<.05)($ TABLA 2).

\section{Tabla 2}

Frecuencias, porcentajes y prueba $\mathrm{x} 2$ sobre gastos generales del estudiante

\begin{tabular}{|c|c|c|c|c|c|c|c|}
\hline \multirow[t]{2}{*}{ Variable } & \multirow[t]{2}{*}{ Opciones de respuesta } & \multicolumn{2}{|c|}{ Pretest } & \multicolumn{2}{|c|}{ Postest } & \multirow[t]{2}{*}{$\mathrm{x}^{2}$} & \multirow[t]{2}{*}{$\mathbf{p}$} \\
\hline & & $\mathbf{f}$ & $\%$ & f & $\%$ & & \\
\hline \multirow{3}{*}{$\begin{array}{l}\text { Gastos } \\
\text { generales }\end{array}$} & Ordenador, juegos online... & 43 & 16.8 & 23 & 13.8 & \multirow{3}{*}{333.23} & \multirow{3}{*}{.00} \\
\hline & Móvil, aplicaciones... & 42 & 10.4 & 30 & 9.7 & & \\
\hline & Máquinas tragaperras, póker, ruleta. & 4 & 1.4 & 2 & 0.5 & & \\
\hline \multirow{7}{*}{$\begin{array}{l}\text { Gasto mensual } \\
\text { de móvil }\end{array}$} & Nada & 78 & 12.2 & 190 & 29.7 & \multirow{7}{*}{116,57} & \multirow{7}{*}{.00} \\
\hline & Menos de 10 euros & 123 & 19.2 & 303 & 47.4 & & \\
\hline & Entre 10 y 30 euros & 168 & 26.3 & 108 & 16.9 & & \\
\hline & Entre 30 y 60 euros & 3 & 11.4 & 28 & 4.4 & & \\
\hline & Entre 60 y 80 euros & 6 & 0.9 & 0 & 0 & & \\
\hline & Más de 80 euros & 10 & 1.6 & 0 & 0 & & \\
\hline & No lo sé & 179 & 28.0 & 8 & 1.3 & & \\
\hline
\end{tabular}

Nota: Chi cuadrado (x2); ${ }^{\star} \mathrm{p} \leq .05$

En lo relativo al uso del ordenador con conectividad a Internet, se debe de destacar el decrecimiento de la usabilidad de dichas computadoras para jugar a video juegos (pretest $=16.3 \%$, postest $=3.6 \%$ ) o realizar apuestas online una vez llevado a cabo el programa (pretest=5.3\%, 
postest=0.3\%). A su vez, como se observa en la tabla 3, en el postest aumenta el uso de los ordenadores para la búsqueda de información que les permite realizar trabajos escolares (pretest $=5.6 \%$, postest $=23.1 \%)(x 2=45.34, p<.05)$. Con respecto a la frecuencia en el uso del ordenador, se reduce sustancialmente una vez que los participantes intervinieron en el programa $(\mathrm{X} 2=289.77, \mathrm{p}<.05)$. Por último, después de la intervención, los participantes eran más conscientes de la dependencia que sentían de todo tipo de aparatos tecnológicos con conexión a red $(x 2=16.23, p<.05)$.

\section{Tabla 3}

Frecuencias, porcentajes y prueba 22 en relación al uso de ordenadores con conectividad a red

\begin{tabular}{|c|c|c|c|c|c|c|c|}
\hline \multirow[t]{2}{*}{ Variable } & \multirow[t]{2}{*}{ Opciones de respuesta } & \multicolumn{2}{|c|}{ Pretest } & \multicolumn{2}{|c|}{ Postest } & \multirow[t]{2}{*}{$x^{2}$} & \multirow[t]{2}{*}{$\mathbf{p}$} \\
\hline & & $\mathrm{f}$ & $\%$ & $\mathbf{f}$ & $\%$ & & \\
\hline \multirow[t]{7}{*}{$\begin{array}{l}\text { Usos del ordenador } \\
\text { con acceso a } \\
\text { Internet }\end{array}$} & $\begin{array}{l}\text { Chatear, whatsApp, Facebook } \\
\text { Twitter, Instagram, mandar } \\
\text { correos... }\end{array}$ & 429 & 67.1 & 434 & 68.1 & & \\
\hline & Hacer trabajos para el Instituto & 36 & 5.6 & 147 & 23.1 & & \\
\hline & Jugar a videojuegos online & 104 & 16.3 & 23 & 3.6 & & \\
\hline & Jugar al póker, ruleta...online & 34 & 5.3 & 2 & 0.3 & 45.34 & .00 \\
\hline & $\begin{array}{l}\text { Bajarme música, películas, } \\
\text { programas... }\end{array}$ & 24 & 3.8 & 25 & 3.9 & & \\
\hline & $\begin{array}{l}\text { Navegar buscando fotos o } \\
\text { información que me interesa... }\end{array}$ & 10 & 1.6 & 6 & 0.9 & & \\
\hline & $\begin{array}{l}\text { Hacer compras online, usar } \\
\text { Wallapop... }\end{array}$ & 2 & 0.3 & 0 & 0 & & \\
\hline \multirow{8}{*}{$\begin{array}{l}\text { Frecuencia uso } \\
\text { Internet }\end{array}$} & Todos los días, 5 horas o más & 181 & 28.3 & 73 & 11.5 & & \\
\hline & Todos los días, 4 a 5 horas. & 65 & 10.2 & 41 & 6.4 & & \\
\hline & Todos los días, 3 a 4 horas & 105 & 16.5 & 83 & 13.0 & & \\
\hline & Todos los días, 2 a 3 horas & 92 & 14.4 & 77 & 12.1 & & \\
\hline & $\begin{array}{l}\text { Todos los días, menos de } 2 \\
\text { horas }\end{array}$ & 112 & 17.6 & 138 & 21.6 & 289.77 & .00 \\
\hline & Entre 3 y 5 veces a la semana & 29 & 4.5 & 103 & 16.1 & & \\
\hline & Muy de vez en cuando & 50 & 7.8 & 70 & 11.0 & & \\
\hline & Nunca & 3 & 0.5 & 52 & 8.1 & & \\
\hline \multirow{2}{*}{$\begin{array}{l}\text { Reconocimiento de } \\
\text { frecuencia alta de } \\
\text { uso de Internet }\end{array}$} & Sí & 82 & 12.8 & 350 & 54.8 & & \\
\hline & No & 555 & 86.9 & 287 & 45.1 & 16.23 & .00 \\
\hline
\end{tabular}

Nota: Chi cuadrado $(\mathrm{X} 2) ;{ }^{*} \mathrm{p} \leq .05$

Tras la intervención, los participantes aseguraron haber reducido el juego y las apuestas vía online a través del teléfono móvil (pretest $=9.8 \%$, postest $=0.8)(x 2=80.96, p<.05)$. En cuanto a la frecuencia de su uso, el alumnado reconocía pasar demasiado tiempo con el móvil manifestándose nuevamente la eficiencia del programa de intervención para la concienciación de la población estudiantil $(x 2=35.88, p<.05)($ Tabla 4$)$.

Finalmente, se analizaron cambios en la conducta, adquisición de terminología y valoración el programa por parte de los participantes una vez llevada a cabo la intervención. Se puede observar en la tabla 5, como la vulnerabilidad del alumnado para dejarse convencer por terceras personas se redujo tras el programa de intervención $(x 2=37.13, p<.05)$. Este aspecto es de suma importancia a la hora de prevenir adicciones producidas por la ausencia de la capacidad de negación. 


\section{Tabla 4}

Frecuencias, porcentajes y prueba $x 2$ en referencia al uso del móvil

\begin{tabular}{|c|c|c|c|c|c|c|c|}
\hline \multirow[t]{2}{*}{ Variable } & \multirow[t]{2}{*}{ Opciones de respuesta } & \multicolumn{2}{|c|}{ Pretest } & \multicolumn{2}{|c|}{ Postest } & \multirow[t]{2}{*}{$x^{2}$} & \multirow[t]{2}{*}{$\mathbf{p}$} \\
\hline & & f & $\%$ & $\mathbf{f}$ & $\%$ & & \\
\hline \multirow[t]{6}{*}{ Usos de móvil } & Llamadas & 15 & 2.3 & 63 & 9.9 & & \\
\hline & Mensajes (WhatsApp, Line...) & 444 & 69.5 & 430 & 67.3 & & \\
\hline & Navegación & 88 & 13.8 & 97 & 15.2 & 80.96 & .00 \\
\hline & Agenda & 6 & 0.9 & 1 & 0.2 & & \\
\hline & Juegos & 60 & 9.4 & 5 & 0.8 & & \\
\hline & Otros & 24 & 3.8 & 41 & 6.4 & & \\
\hline \multirow{2}{*}{$\begin{array}{l}\text { Reconocimiento de } \\
\text { frecuencia alta de } \\
\text { uso de móvil }\end{array}$} & Sí & 299 & 46.8 & 488 & 76.4 & & \\
\hline & No & 338 & 52.9 & 149 & 23.4 & 35.88 & .00 \\
\hline
\end{tabular}

Nota: Chi cuadrado (X2); ${ }^{*} p \leq .05$

En cuanto a la adquisición de conceptos nuevos, como son el caso de los términos adicción y juego patológico, en el postest hubo un mayor porcentaje de alumnos los cuales determinaron que estos dos conceptos hacían referencia a enfermedades (adicción $=71,7 \%$; juego patológico $=82,4 \%$ ), a diferencia de en el pretest donde un mayor porcentaje respondían equívocamente diciendo que eran algún tipo de vicio (adicción=66,7\%; juego patológico=14,3\%) o simplemente confesaban no saber la respuesta (adicción $=4,7 \%$; juego patológico $=43 \%$ ) (Concepto de adiciones: $x 2=36.30, p<.05$; concepto de juego patológico: $x 2=40.56, p<.05$ ). Por último, el alumnado pasó de pensar que la intervención sería un "rollo" de mayores o que le permitiría perder clase, a concluir que este tipo de acciones educativas pueden ser fructíferas para ellos $\left(x^{2}=29.94, p<.05\right)$.

Tabla 5.

Frecuencia, porcentaje y prueba de $x^{2}$ sobre cambios de conducta, adquisición de terminología y valoración del programa de intervención

\begin{tabular}{llrrrrrr}
\hline \multicolumn{1}{c}{ Variable } & Opciones de respuesta & \multicolumn{2}{c}{ Pretest } & \multicolumn{2}{c}{ Postest } & X2 & p \\
& & $\mathbf{f}$ & $\%$ & $\mathbf{f}$ & $\%$ & & \\
\hline \multirow{2}{*}{ Dejarse influenciar } & Sí & 213 & 33.4 & 5 & 0.8 & & \\
& No & 200 & 31.4 & 435 & 68.3 & 37.13 & .00 \\
& A veces & 224 & 35.2 & 197 & 30.9 & & \\
$\begin{array}{l}\text { Conocimiento del } \\
\text { propio uso de }\end{array}$ & Sí & & & & & & \\
$\begin{array}{l}\text { Internet y móvil por } \\
\text { parte de los padres }\end{array}$ & No & 431 & 67.7 & 538 & 84.5 & 45.91 & .00 \\
\hline
\end{tabular}




\begin{tabular}{|c|c|c|c|c|c|c|c|}
\hline \multirow{4}{*}{$\begin{array}{l}\text { ¿Qué es la } \\
\text { adicción? }\end{array}$} & Un vicio & 426 & 66.7 & 143 & 22.4 & \multirow{4}{*}{36.30} & \multirow{4}{*}{.00} \\
\hline & Una enfermedad & 155 & 24.3 & 457 & 71.7 & & \\
\hline & Una mala conducta & 26 & 4.1 & 29 & 4.6 & & \\
\hline & No lo sé & 30 & 4.7 & 8 & 1.3 & & \\
\hline \multirow{4}{*}{$\begin{array}{l}\text { ¿Qué es el juego } \\
\text { patológico? }\end{array}$} & $\begin{array}{l}\text { Un vicio que consiste en } \\
\text { que la persona juega } \\
\text { mucho }\end{array}$ & 91 & 14.3 & 35 & 5.5 & \multirow{4}{*}{40.56} & \multirow{4}{*}{.00} \\
\hline & $\begin{array}{l}\text { Una afición, es decir, una } \\
\text { persona a la que le gusta } \\
\text { mucho jugar }\end{array}$ & 37 & 5.8 & 17 & 2.7 & & \\
\hline & $\begin{array}{l}\text { Una enfermedad que } \\
\text { consiste en ser adicto al } \\
\text { juego }\end{array}$ & 235 & 36.9 & 525 & 82.4 & & \\
\hline & No lo sé & 274 & 43.0 & 60 & 9.4 & & \\
\hline \multirow{4}{*}{$\begin{array}{l}\text { Opinión sobre la } \\
\text { intervención }\end{array}$} & $\begin{array}{l}\text { Que son un rollo de } \\
\text { mayores }\end{array}$ & 280 & 44.0 & 7 & 1.1 & \multirow{4}{*}{29.94} & \multirow{4}{*}{.00} \\
\hline & $\begin{array}{l}\text { Que son útiles y nos } \\
\text { enseña a evitar algunos }\end{array}$ & 132 & 20.7 & 570 & 89.5 & & \\
\hline & $\begin{array}{l}\text { problemas } \\
\text { Bien, porque nos libramos } \\
\text { de clase }\end{array}$ & 200 & 31.4 & 17 & 2.7 & & \\
\hline & $\begin{array}{l}\text { Me gustan porque son } \\
\text { entretenidas }\end{array}$ & 25 & 3.9 & 43 & 6.8 & & \\
\hline
\end{tabular}

Nota: Nota: Chi cuadrado ( $\mathrm{x} 2) ;{ }^{*} \mathrm{p} \leq .05$

\section{Discusión y conclusiones}

El objetivo de este estudio fue analizar la eficacia del programa para la prevención de adicciones a Internet, móvil, juegos y apuestas online "Cubilete" sobre la población adolescente en las horas de tutoría. Los resultados obtenidos muestran un claro efecto positivo del mismo sobre la población participante en lo referente al uso excesivo de las TIC, así como para la disminución de hábitos negativos relacionados con el juego de azar y las apuestas online.

La frecuencia en el uso de las TIC en los estudiantes de Educación Secundaria y Bachillerato es realmente alta antes de llevar a cabo el programa "Cubilete". Esto refleja la gran influencia que tienen las tecnologías sobre esta población joven (Punamäki, Wallenius, Nygård, Saarni, y Rimpelä, 2007; Festl, Scharkow, y Quandt, 2013; Glušac, Makitan, Karuović, Radosav, y Milanov, 2015). Una explicación del crecimiento del uso de las TIC puede ser el mayor protagonismo que el adolescente tiene dentro del núcleo familiar así como en la toma de decisiones en lo referente al manejo de más dinero para sus propios gastos (Denegri Coria, Lara Arzola, Córdova Cares, y Del Valle Rojas, 2008). Esos gastos, según lo observado en los resultados del estudio, están en mayor medida orientados a elementos de uso cotidiano como ropa, bebidas, alimentación, etc., aunque seguido de gastos en aplicaciones de ordenador o móvil, juegos online y demás gastos dedicados a las TIC. De acuerdo con lo mostrado en esta investigación, más de la mitad del alumnado (54,9\%), una vez realizado el programa de intervención, reconoce tener una frecuencia alta. Sin embargo, en un estudio trasversal en el que a los estudiantes no se les hace participes de programas de prevención hacía el uso abusivo de Internet, sólo el $15 \%$ de la muestra total 
reconocía usar la red diariamente (Viñas Poch et al., 2002). Esto muestra un evidente signo de negación por parte de la población adolescente, la cual no es consciente de los riesgos procedentes al uso abusivo de las TIC (Encinas Labrador, Requesens Moli, y Helguera Fuentes, 2011).

El uso que se le da a las TIC, principalmente cuando éstas están conectadas a Internet, es punto de inflexión de expertos de la materia preocupados por los efectos adversos que estas pueden provocar sobre la población joven. Las adicciones a su uso así como comportamientos obsesivos a juegos virtuales y apuestas online son algunas de las consecuencias generadas por un manejo nocivo de las mismas. Como se puede observar en los resultados de esta investigación, las acciones más repetidas por parte del alumnado en cuanto al uso de las TIC conectadas a red son las referentes a las telecomunicaciones (chatear, whatsApp, Facebook Twitter, Instagram, mandar correos, etc). Como afirma Salehan y Negahban (2013), el uso en exceso de aplicaciones de redes sociales en línea es un predictor claro de adicción. Esas acciones van seguidas de la descarga de juegos online y el depósito en apuestas virtuales. Estos mismos resultados se obtienen en la investigación dirigida por Sarabia Gonzalvo, Herrero Fernández, y Estévez Gutiérrez, (2014) quienes determinan que la aparición violenta de las TIC, la normalización de los juegos de azar y las apuestas online, así como la facilidad de acceso de los mismos, constituye una de las razones principales de su incremento entre los jóvenes. Por otro lado, un buen uso de las TIC orientado al mundo académico puede favorecer el aprendizaje. Capacidades como la memoria de trabajo o la habilidad de tomar decisiones rápidas se ven mejoradas a través de un correcto uso de Internet (Arab y Díaz, 2015) o incluso de juegos virtuales (Ruiz-Ariza, Casuso, Suarez-Manzano, y Martínez-López, 2018). Además, según los autores anteriormente citados, los adolescentes llegan a concienciarse del efecto positivo del uso de Internet en las tareas escolares. Estos resultados entran en concordancia con los obtenidos en nuestra investigación una vez llevada a cabo la intervención.

Las intervenciones destinadas a la reducción de comportamientos relacionados con el juego patológico en cualquier contexto son realmente necesarias. Como se ha podido observar en esta investigación, la reducción de este tipo de práctica nociva para la salud mental del alumnado ha sido significativa tras la intervención. Estos resultados se asemejan a los obtenidos por GarciaCaballero et al. (2018) quienes tras 6 meses de intervención y 6 de seguimiento con pacientes con problemas de juego consiguieron disminuir de manera significativa el trastorno por juego patológico de 6.72 a 1.33 puntos obtenidos en el cuestionario Screen for Gambling Problems (NODS). Del mismo modo, Rizeanu (2014) llevó a cabo un modelo de terapia cognitivo-conductual para pacientes con trastorno de juego patológico y sus derivados como la ansiedad o la depresión. Tras la intervención y después de un periodo de 3 meses de seguimiento, en un $74,6 \%$ de los participantes, que terminaron el tratamiento, desapareció el trastorno por juego patológico, además de reducirse considerablemente sus niveles de ansiedad y/o depresión. En la edad adolescentes, a pesar del reducido número de intervenciones encontradas en contextos escolares, estudios como los de Walther, Hanewinkel, y Morgenstern (2013), demostraron la efectividad, incluso en periodos cortos de tratamiento en los estudiantes, en la reducción de rutinas nocivas sobre el juego. Además, Donati, Primi, y Chiesi (2014) mostró como un programa basado en técnicas de capacitación para la entrega de los contenidos educativos (actividades con generadores de recompensa, diapositivas de Powerpoint, videos y discusiones en grupo) mejoraban el conocimiento correcto sobre el juego, reducían los conceptos erróneos, la percepción de la rentabilidad del juego y el pensamiento supersticioso.

Uno de los principales problemas a los que los adolescentes se enfrentan, en cuanto a trastornos relacionados con juegos de azar y apuestas virtuales, es al desconocimiento de términos como adicción o juego patológico. Como ha puesto de manifiesto este estudio, los participantes antes de la intervención prácticamente desconocían el significado real de dichos términos. Sin embargo, una vez llevado a cabo el programa, aumenta considerablemente la comprensión de los mismos. En esa misma línea, Martín del Río y Cortés Tomás (2004) destacan la importancia de formar al alumnado en esa terminología para una mayor prevención de ese tipo de trastorno. Markez (2015) a su vez, expone cómo la atención integral, así como la acción preventiva ante el conocimiento de terminología y semántica relacionada con adicciones en esa 
edad, promueven un mayor conocimiento y por tanto una reducción de pacientes que padecen trastornos por adicciones o juego patológico.

Una de las limitaciones de este estudio ha sido la falta de un periodo de seguimiento de los participantes una vez terminada la intervención, lo que le podía haber dado un carácter más efectivo a la misma. Además, un grupo control que no hubiese participado en la intervención hubiese dado potencialidad al método experimental del estudio. Como fortaleza se puede destacar la amplia muestra participante, la edad que abarca el estudio, dada su dificultad a la hora de intervenir sobre los individuos en este periodo, y la diversidad en cuanto al carácter sociodemográfico de la muestra.

En consecuencia, este estudio pone de manifiesto la efectividad de las intervenciones llevadas a cabo dentro de contextos escolares en lo referente al uso de TIC así como para disminución de adicciones a juegos de azar y apuestas online. Programas de intervención de estas características permiten al alumnado ser más conscientes de la problemática planteada reduciendo así su frecuencia. Utilizar las horas de tutoría como medio para hacer partícipes al alumnado en programas de intervención que traten problemas surgidos en estas edades es una herramienta de gran valor educativo, psicológico y social. En esta línea, Weiss (2016) subraya como uno de los objetivos principales a llevar a cabo durante las horas de tutoría en la Educación Secundaria, es la orientación para la vida, que incluye dar respuesta a cualquier tipo de problema que sufra el alumnado. De este modo, se manifiestan como futuras líneas de intervención, promover este tipo de programas educativos en un mayor número de centros escolares durante las horas de tutoría como una herramienta eficaz para la prevención de adicciones al uso de las TIC, reducción del juego patológico y participación en apuestas online. Se precisan más programas y/o tratamientos destinados a la población adolescente en contextos educativos con la finalidad de alcanzar un mayor grado de prevención de este tipo de conductas y trastornos nocivos para la salud.

\section{Referencias bibliográficas}

Allami, Y., y Vitaro, F. (2015). Pathways model to problem gambling: clinical implications for treatment and prevention among adolescents. The Canadian Journal of Addiction, 6(2), 1319.

Retrieved

from:

https://journals.Iww.com/cja/Abstract/2015/09000/Pathways_Model_to_Problem_Gambling Clinical.3.aspx

Arab, L. E., y Díaz, G. A. (2015). Impacto de las redes sociales e internet en la adolescencia: aspectos positivos y negativos. Revista Médica Clínica Las Condes, 26(1), 7-13. doi: 10.1016/J.RMCLC.2014.12.001

Beranuy, M., Chamarro, A., Graner, C., y Carbonell, X. (2009). Validación de dos escalas breves para evaluar la adicción a Internet y el abuso de móvil. Psycothema, 21(3), 480-485. Recuperado de: http://www.psicothema.com/psicothema.asp?id=3658

Carbonell, X. (2014). La adicción a los videojuegos en el DSM-5. Adicciones, 26(2), 91. doi: 10.20882/adicciones.10

Caro Amada, C., y Plaza de la Hoz, J. (2016). Intervención educativa familiar y terapia sistémica en la adicción adolescente a internet: fundamentación teórica. Revista Española de Orientación y Psicopedagogía (REOP), 27(1), 99-113. 
Castellana-Rosell, M., Sánchez Carbonell, X., Graner-jordana, C., y Beranuy-Fargues, M. (2007). El adolescente ante las tecnologías de la información y la comunicación: Internet, móvil y videojuegos. Papeles del Psicólogo (Vol. 28). Consejo General de Colegios Oficiales de Psicólogos. Recuperado de: http://www.redalyc.org/html/778/77828306/

Chak, K., y Leung, L. (2004). Shyness and Locus of Control as Predictors of Internet Addiction and Internet Use. Cyberpsychology y Behavior, 7(5). Retrieved from: www.netaddiction.com

Chassiakos, Y. L. R., Radesky, J., Christakis, D., Moreno, M. A., y Cross, C. (2016). Children and adolescents and digital media. Pediatrics, 138(5), e20162593. doi: 10.1542/peds.2016-2593

Cía, A. H. (2013). Las adicciones no relacionadas a sustancias (DSM-5, APA, 2013): un primer paso hacia la inclusión de las Adicciones Conductuales en las clasificaciones categoriales vigentes. Revista de Neuro-Psicatria, 76(4), 210-217. doi: 10.20453/rnp.v76i4.1169

Ciarrochi, J., Parker, P., Sahdra, B., Marshall, S., Jackson, C., Gloster, A. T., y Heaven, P. (2016). The development of compulsive internet use and mental health: A four-year study of adolescence. Developmental Psychology, 52(2), 272-283. doi: 10.1037/dev0000070

Delfabbro, P., King, D. L., y Derevensky, J. L. (2016). Adolescent Gambling and Problem Gambling: Prevalence, Current Issues, and Concerns. Current Addiction Reports, 3(3), 268274. doi: $10.1007 / s 40429-016-0105-z$

Denegri Coria, M., Lara Arzola, M. Á., Córdova Cares, G., y Del Valle Rojas, C. (2008). Prácticas de ahorro y uso del dinero en pre adolescentes (tweens) chilenos. Universum (Talca), 23(1), 24-38. doi: 10.4067/S0718-23762008000100003

Donati, M. A., Primi, C., y Chiesi, F. (2014). Prevention of Problematic Gambling Behavior Among Adolescents: Testing the Efficacy of an Integrative Intervention. Journal of Gambling Studies, 30(4), 803-818. doi: 10.1007/s10899-013-9398-1

Dowling, N. A., Merkouris, S. S., y Lorains, F. K. (2016). Interventions for comorbid problem gambling and psychiatric disorders: Advancing a developing field of research. Addictive Behaviors, 58, 21-30. doi: 10.1016/J.ADDBEH.2016.02.012

Ellison, L. J., Vale, K., y Ladouceur, R. (2017). Disordered Gambling: The Effects of a Brief, Online Cognitive Intervention in an Australian Sample. OALib Journal, 4(1), 1-12. doi: 10.4236/oalib.1103288

Encinas Labrador, E., Requesens Moli, A., y Helguera Fuentes, M. (2011). Guía para padres y educadores sobre el uso seguro de Internet, videojuegos y móviles. Madrid: Fundación Gaudium. Retrieved from: http://www.juntadeandalucia.es/educacion/webportal/ishareservlet/content/692e8985-b99c-4d90-8f94-208770db80a7

Estévez Gutiérrez, A., Herrero Fernández, D., Sarabia Gonzalvo, I., y Jáuregui Bilbao, P. (2014). El papel mediador de la regulación emocional entre el juego patológico, uso abusivo de Internet y videojuegos y la sintomatología disfuncional en jóvenes y adolescentes. Adicciones, 26(4), 282. doi: 10.20882/adicciones.26

Festl, R., Scharkow, M., y Quandt, T. (2013). Peer Influence, Internet use and Cyberbullying: A Comparison of Different Context Effects among German Adolescents. Journal of Children and Media, 7(4), 446-462. doi: 10.1080/17482798.2013.781514

Gainsbury, S., y Blaszczynski, A. (2011). Online self-guided interventions for the treatment of problem gambling. International Gambling Studies, 11(3), 289-308. doi: 10.1080/14459795.2011.617764

Garcia-Caballero, A., Torrens-Lluch, M., Ramírez-Gendrau, I., Garrido, G., Vallès, V., y Aragay, N. (2018). Eficacia de la intervención Motivacional y la Terapia Cognitivo-conductual para el 
tratamiento del Juego Patológico. Adicciones, 30(3), 219. doi: 10.20882/adicciones.965

Glušac, D., Makitan, V., Karuović, D., Radosav, D., y Milanov, D. (2015). Adolescents' informal computer usage and their expectations of ICT in teaching - Case study: Serbia. Computers y Education, 81, 133-142. doi: 10.1016/J.COMPEDU.2014.10.006

Gray, H. M., LaPlante, D. A., y Shaffer, H. J. (2012). Behavioral characteristics of Internet gamblers who trigger corporate responsible gambling interventions. Psychology of Addictive Behaviors, 26(3), 527-535. doi: 10.1037/a0028545

Group, E. (2016). ESPAD Report 2015 Results from the European School Survey Project on Alcohol and Other Drugs. Lisbon, Portugal: European Monitoring Centre on Drugs and Drugs Addiction.

Halen, L., y Guan, S. (2015). Screen time and sleep among school-aged children and adolescents: A systematic literature review. Sleep Medicine Reviews, 21, 50-58. doi: 10.1016/j.smrv.2014.07.007

Hysing, M., Pallesen, S., Stormark, K., Jakobsen, R., Lundervold, A., y Sivertsen, B. (2015). Sleep and use of electronic devices in adolescence: results from a large population-based study. BMJ Open, 5(1), e006748. doi: 10.1136/bmjopen-2014-006748

Ladouceur, R., Sylvain, C., Boutin, C., y Doucet, C. (2002). Understanding and treating the pathological gambler. West Sussex, England: John Wiley y Sons. Retrieved from: https://www.wiley.com/en-us/Understanding+and+Treating+the+Pathological+Gambler-p9780470843789

Markez, I. (Coord. . (2015). Adicciones: Conocimiento, atención integral y acción preventiva. Madrid: Asociación Española de Neuropsiquiatría de Salud Mental. Retrieved from: https://osalde.org/wp-content/uploads/2017/02/2015-Adicciones-56-AEN.pdf

Martín del Río, B., y Cortés Tomás, M. (2004). Acceso a la documentación y formación en juego patológico. Salud Y Drogas, 4(2). Recuperado de: http://www.redalyc.org/html/839/83940207/

Oberst, U., Wegmann, E., Stodt, B., Brand, M., y Chamarro, A. (2017). Negative consequences from heavy social networking in adolescents: The mediating role of fear of missing out. Journal of Adolescence, 55, 51-60. Doi: 10.1016/J.ADOLESCENCE.2016.12.008

Ólafsson, K., Livingstone, S., y Haddon, L. (2014). Children's use of online technologies in Europe: a review of the European evidence base (revised edition). London, UK: EU Kids Online.

Park, Y. J. (2015). My whole world's in my palm! The second-level divide of teenagers' mobile use and skill. New Media y Society, 17(6), 977-995. doi: 10.1177/1461444813520302

Pérez de Albéniz-Garrote, G, Medina Gómez, B., y Rubio Rubio, L. (2019). Influencia de la impulsividad y de la búsqueda de sensaciones en el consumo precoz de cannabis. Diferencias de género y orientaciones para la prevención. Revista Española de Orientación y Psicopedagogía, 30(1), 27-40.

Pontes, H. M., y Griffiths, M. D. (2015). Measuring DSM-5 internet gaming disorder: Development and validation of a short psychometric scale. Computers in Human Behavior, 45, 137-143. doi: 10.1016/J.CHB.2014.12.006

Potenza, M. N. (2013). Neurobiology of gambling behaviors. Current Opinion in Neurobiology, 23(4), 660-667. doi: 10.1016/j.conb.2013.03.004

Punamäki, R.-L., Wallenius, M., Nygård, C.-H., Saarni, L., y Rimpelä, A. (2007). Use of information and communication technology (ICT) and perceived health in adolescence: The role of sleeping habits and waking-time tiredness. Journal of Adolescence, 30(4), 569-585. doi: 10.1016/J.ADOLESCENCE.2006.07.004 
Rizeanu, S. (2014). The Efficacy of Cognitive-behavioral Intervention in Pathological Gambling Treatment. Procedia - Social and Behavioral Sciences, 127, 626-630. doi: 10.1016/J.SBSPRO.2014.03.324

Rodríguez Torres, A. Prats, M. Á., Oberst, U., y Carbonell, X. (2018). Diseño y aplicación de talleres educativos para el uso saludable de internet y redes sociales en la adolescencia: descripción de un estudio piloto. Pixel-Bit, 52, 111-124. doi: 10.12795/pixelbit.2018.i52.08

Ruiz-Ariza, A., Casuso, R. A., Suarez-Manzano, S., y Martínez-López, E. J. (2018). Effect of augmented reality game Pokémon GO on cognitive performance and emotional intelligence in adolescent young. Computers $y$ Education, 116, 49-63. doi: 10.1016/J.COMPEDU.2017.09.002

Ruiz-Olivares, R., Lucena, V., Pino, M. J., y Herruzo, J. (2010). Análisis de comportamientos relacionados con el uso/abuso de Internet, teléfono móvil, compras y juego en estudiantes universitarios. Adicciones, 22(4), 301-310. Recuperado de: https://helvia.uco.es/bitstream/handle/10396/11554/301-310 herruzo. pdf? sequence $=1$ yisAllowed $=y$

Salehan, M., y Negahban, A. (2013). Social networking on smartphones: When mobile phones become addictive. Computers in Human Behavior, 29(6), 2632-2639. doi: 10.1016/J.CHB.2013.07.003

Sarabia Gonzalvo, I., Herrero Fernández, D., y Estévez Gutiérrez, A. (2014). Situación actual del juego con dinero en jóvenes y adolescentes. Revista Española de Drogodependencias, 39(3), 57. Recuperado de: http://roderic.uv.es/handle/10550/47655

Symons, K., Ponnet, K., Walrave, M., y Heirman, W. (2017). A qualitative study into parental mediation of adolescents' internet use. Computers in Human Behavior, 73(C), 423-432. doi: 10.1016/j.chb.2017.04.004

Toneatto, T. (2016). Single-session interventions for problem gambling may be as effective as longer treatments: Results of a randomized control trial. Addictive Behaviors, 52, 58-65. Doi: 10.1016/J.ADDBEH.2015.08.006

Uchuypoma Canchumani, D. (2017). Juegos online: una mirada desde el juego patológico. Hamut'ay, 4(2), 55-64. doi: 10.21503/hamu.v4i2.1472

Viñas Poch, F., Ferrer, J. J., Villar Hoz, E., Caparros Caparros, B., Pérez Guerra, I., y Cornella Canals, M. (2002). Internet y psicopatología: las nuevas formas de comunicación y su relación con diferentes índices de psicopatología. Clínica Y Salud, 13(3), 235-256. Recuperado de: http://www.redalyc.org/pdf/1806/180618090001.pdf

Walther, B., Hanewinkel, R., y Morgenstern, M. (2013). Short-Term Effects of a School-Based Program on Gambling Prevention in Adolescents. Journal of Adolescent Health, 52(5), 599605. doi: 10.1016/J.JADOHEALTH.2012.11.009

Weiss, E. (2016). La apropiación de una innovación. La hora de Orientación y tutoría en escuelas secundarias. Revista Electrónica de Investigación Educativa, 18(2), 1-14. Recuperado de: http://www.scielo.org.mx/scielo.php?pid=S1607$40412016000200001 y$ script=sci_arttextytlng=en

Yan, Z. (2017). Child and Adolescent Use of Mobile Phones: An Unparalleled Complex Developmental Phenomenon. Child Development, 89(1), 5-16. doi: 10.1111/cdev.12821 
Fecha de entrada: 08 noviembre 2019 Fecha de revisión: 29 febrero 2020

Fecha de aceptación: 07 marzo 2020 